\title{
Interrupted Versus Continuous Suture Technique in End-to-End Stentless Biliary Reconstruction
}

\author{
IBRAHIM ABOULFOTOH MOHAMMED, M.D. \\ The Department of General Surgery, Faculty of Medicine, Al-Azhar University
}

\begin{abstract}
Background: A wide variety of techniques to restore biliary-enteric continuity after biliary injuries, the biliary tree is most commonly anastomosed to the jejunum; either as (Roux-en-Y or a simple loop anastomosis) or less commonly to the duodenum. The anastomosis may be performed to the bile duct, common hepatic duct, 1 st or 2 nd order hepatic duct branches, or to the gallbladder. Anastomoses may be performed in an end-to-side or side-to-side fashion, using continuous or interrupted sutures. Stents may be deployed across the anastomosis.
\end{abstract}

Aim of Study: Biliary strictures and injuries; present a surgical technical challenge for Hepato-biliary surgeons. High biliary strictures are difficult to treat and re-stenosis rates reported of $10-30 \%$ following corrective repair, Complication rates; mostly due to inflammation, ischemia and tension at the anastomosis site. A combined prospective and retrospective study to evaluate the outcome of bile duct reconstruction using a continuous and interrupted suture technique for anastomosis. A combined prospective and retrospective study to evaluate the outcome of bile duct reconstruction using a continuous and interrupted suture technique for anastomosis..

Patients and Methods: A total number of 30 patients presented with biliary injuries, biliary strictures or stenosis permitted for surgical interventions in a General Surgical Department of Al-Azhar University Hospitals from February 2015 to May 2019, 15 patients were performed by continuous suture as (Group A), and 15 patents performed by interrupted suture technique as (Group B). Data and Statistical Analysis: Data on our series of bile duct anastomosis with either distal biliary duct or small bowel performed including: Surgical procedure, diagnosis, co-morbidities, peri-operative morbidity, and clinical symptoms after repair, liver function tests and the need for surgical or radiological interventions following surgery were evaluated as a prospectively collected on an institutionally approved electronic database. Non-parametric data were described as median and range and parametric data as average and standard deviation. The outcome measures were assessed: peri-operative morbidity, clinical symptoms after repair, liver function tests and the need for surgical or radiological interventions following surgery.

Correspondence to: Dr. Ibrahim Aboulfotoh Mohammed, The Department of General Surgery, Faculty of Medicine, Al-Azhar University
Results: End-to-ends Biliary reconstruction, Hepatico-, Choledocho jejnostomy anastomoses were performed. In (Group A) 15 patients underwent biliary reconstruction using the continuous suture technique. The median operative time was 140 minutes (range 60-300 minutes). Post-operative morbidity was present in three patients $(12.5 \%)$, shows postoperative biliary leak, wound sepsis, post-operative fever in sequence. In 14 (98.25\%) of the 15 patients, there has been no recurrence of jaundice, cholangitis or biliary dilatation on abdominal ultrasonography and liver function tests improved and have returned to normal without further surgical or radiological intervention. Out of anastomoses, there were two patients in (group A) who developed leakage, and 3 patients who developed a stricture for an incidence of $1.75 \%$ and $3.25 \%$ respectively. Our overall biliary complication rate was $4 \%$, which is lower than previously reported.

Conclusions: A continuous suture technique of biliary anastomoses results in an extremely low incidence of common significant complications.

Key Words: Biliary reconstruction - Risk factors and complications.

\section{Introduction}

RECONSTRUCTION of the biliary tracked is required in several clinical situations, including resection for benign or malignant biliary strictures, intra-operative biliary injury, and liver transplantation [1]

End-to-end ductal anastomosis is a physiologic biliary reconstruction that is commonly used in general surgery, including iatrogenic bile duct injuries [2].

The end-to-end ductal anastomosis has some limitations and cannot be performed in patients with complex vasculo-biliary injuries. Injury of a right hepatic artery is the most frequent type of vasculo-biliary injury. A right hepatic artery injury is the most frequent type of Vasculo-biliary injuries [3]. 
End-to-end ductal anastomosis and Roux-enY Hepatico -jejunostomy are the two most common biliary reconstructions, and the former is the most common in patients who have had liver transplantation, including those with Primary Sclerosing Cholangitis [4]

The traditional technique of Hepaticojejunostomy has been challenged in recent years, by end-to-end biliary reconstruction in these patient groups; however, in patients with iatrogenic bile duct injuries, Hepatico-jejunostomy is performed most frequently [5]

End-to-end ductal anastomosis has many advantages: It is physiologically simpler and associated with fewer post-operative complications than Hepatico -jejunostomy and establishes physiologic bile flow; therefore, (proper digestion and absorption are possible following this procedure), Postoperative diagnostic and therapeutic endoscopic procedures is possible. Despite its advantages, it is not possible to perform end-to-end ductal anastomosis in all patients [6].

Biliary duct reconstruction is a key surgical step in complex Hepato-biliary procedures. Major complications include anastomotic stricture, biliary leak, bleeding, and cholangitis. No definitive data supports the use of interrupted or continues suturing technique [7].

Complications occur mostly due to inflammation, ischemia and tension at the anastomotic site. These complications are ideally reduced through the use of continuous sutures, which induce less scarring, less interruption in blood flow through fewer knot sites, and better suture expansion [8]

General principles:

The main conditions must be met for proper healing of biliary anastomosis:

1- The anastomosed healthy edges; (no inflammation, ischemia or fibrosis).

2- Tension-free anastomosis.

3- Properly vascularized anastomosis [9].

Biliary reconstruction should not be performed in presence of active inflammatory process, patients with ischemia, fibrosis and inflammation within the bile ducts [10].

Multiple techniques for biliary reconstructions have been defined, including End-to-End Choledocho-choledochostomy, and Side-to-Side Choledocho-choledochostomy, Roux-en-Y Hepaticojejunostomy, as well as variations in stenting such as $\mathrm{T}$ tube or internal stenting [11].
Specific similar outcomes between end-to-end Choledocho-choledochostomy and side-to-side Choledocho-choledochostomy. The standard approach for performing end-to-end Choledochocholedochostomy is with an interrupted suture technique [12]

Although; mostly considered the continuous suture technique to be quicker. For end-to-end Choledocho-choledochostomy has not been widely adopted because of concern for higher stricture rates than an interrupted suture technique. However, a comparison of these techniques has not been reported.

Currently, end-to-end ductal anastomosis is the standard biliary reconstruction for in adults. This method is preferable because of an intact sphincter Oddi that can prevent septic cholangitis from ascending infections [13]

Additionally; the procedure facilitates subsequent endoscopic diagnostic and therapeutic procedures in patients with biliary complications. However, end-to-end ductal anastomosis with a small duct ( $<4 \mathrm{~mm}$ in diameter) is associated with a higher risk of biliary strictures than Hepaticojujenostomy [14].

\section{Patients and Methods}

We performed a retrospective review of the prospectively collected database. All patients aged $>22$ years undergoing Biliary Reconstruction in General Surgery Department of Al-Azhar University Hospitals, operated by variable surgical teams, between February 2015 and May 2019, with at least 6 month follow-up were included for the analysis. Patients were divided into two groups on the basis of the procedure.

A primary End-to-End Choledochocholedochostomy was performed for biliary reconstruction. All other types of reconstruction were included, such as choledocho -jejunostomy.

A Written consent Approval was approved by our surgery department protocol Review Board before we performed the study to all patients after a full explanation of the procedure.

Data were collected, using a standardized data collection sheet, including patient age, sex, diagnosis, Data were collected in follow-up on all patients who met the criteria for inclusion until the close of data analysis (10 months after the final Hepatico -jejunostomy anastomosis was performed, 
January 2015). End-points included biliary leak, anastomotic stricture, and time of leak or stricture, re-operation, per-cutaneous or endoscopic interventions for biliary complications had been obtained.

The patient considered has a biliary leak if one of the following criteria is evident:

1 - Bile drainage into intra-operatively placed drains for $>7$ days after Surgery.

2- Post-operative cholangiography shows a leak.

3- Well defined biliary leakage from the wound or abdominal collection.

Re-operation in which a leak was identified.

The patients considered have a biliary stricture if all of the following were present:

1- Clinical manifestation of stricture as; (jaundice and elevated bilirubin or alkaline phosphatase).

2- Cholangiography study demonstrate anastomotic stricture.

In group A (Continuous Suture Stentless Technique): The continuous anastomotic technique was performed with 5.0 PDS suture over a 5 French pediatric feeding tube. Patients in this group; a stent was not used for this anastomosis, a doublearmed suture was first placed at the 6 o'clock position and tied with the knot outside the lumen. Then, each arm was run to the 12 o'clock position in opposite directions and the suture was tied with the final knot outside the bile duct lumen.

In group B (Interrupted Suture Stentless Technique): The interrupted anastomotic technique was per formed with 4 or 5.0 polydioxanone (PDS) sutures over a 5 French pediatric feeding tube with a phalange cut in the distal end to avoid migration of the feeding tube in to the biliary system. The back row sutures were placed and tied such that the knots are in the lumen of the anastomosis. Then, anterior sutures were placed and tied such that the knots are on the outside of the bile duct.

Operative technique:

Division of bile duct:

\section{A- Division of Proximal Part of Extra-hepatic Bile} Duct:

In most pancreato-duodenectomy cases, the bile duct is divided at the middle or upper part of the extra-hepatic bile duct. Encircling the bile duct; is important not to injure the right hepatic artery running just beneath the duct. Before dividing the bile duct, its duodenal side is simply ligated or closed with continuous suture, meanwhile the hepatic side should be occluded with a Bulldog clamp. Electric cautery is used to divide the duct while coagulating the bleeding from the ductal wall. When intra-operative frozen section diagnosis is necessitated, division of the duct should be performed by a surgical blade or scissors to preserve the surgical margin from heat damage by electrocautery. The clamp may be released just before starting the bilio-enteric anastomosis to prevent contaminating the operative field with bile juice.

\section{B- Division of intra-hepatic bile duct:}

During the resection of the hilar or intra-hepatic ducts, the orifice of the bile duct is revealed by dividing the fibrous connective tissue after removing the portal and arterial tributaries from the Glisson's pedicle.

A stay suture with a traumatic needle is placed in the stump of each duct immediately at the time of detection. The suture has to be positioned in the anterior wall of the anastomosis as it used for preserving the intra-luminal space by lifting it up. After diathermy control the bleeding from the bile duct wall, careful inspection is necessary to avoid over-looking tiny branches of the bile ducts such as those arising from the caudate lobe, segment 4 , and/or segment 5 , which joins the hilar bile duct and may cause post-operative biliary leakage.

\section{Anastomotic technique:}

Basic procedure of the anastomosis:

The goal of surgical treatment is to reconstruct the proper bile flow to the alimentary tract. The long-term results depend on the type of performed biliary reconstruction. Two neighboring duct orifices can be joined by two interrupted stitches to prepare them for anastomosis as a common channel.

\section{Sutures of the posterior layer of anastomosis:}

Single-layer interrupted sutures with 4-0 or 50 absorbable mono-filament material are used for the anastomosis. Expert operators can use a limited number of continuous sutures in anastomosis of the extra-hepatic duct.

\section{Technique of end-to-end ductal anastomosis:}

Both proximal and distal ductal stumps should be dissected and approximated without tension. End-to-end ductal anastomosis is recommended for patients when the maximal length loss of the bile duct is $4 \mathrm{~cm}$. The sutured ends have to be healthy, without inflammation or ischemia. The diameter of both anastomosed ends has to be comparable. 
If there was a difference between diameters of anastomosed ends, the narrower end was incised longitudinally in the anterior surface to extend it. End-to-end ductal anastomosis repair was not carried out in bile ducts that were too narrow (diameter $<4 \mathrm{~mm}$ ). The approximating of both ends is possible because of a wide Kocher Maneuver (mobilization of the pancreatic head with the 2 nd $3 \mathrm{rd}$ and ascending part of the duodenum out of the peritoneum). Patients undergoing a first or second bile duct repair can be a candidate for end-to-end ductal anastomosis. Hepatico jejunostomy should be performed in patients who do not satisfy the mentioned criteria.

\section{Suture type:}

Both continuous (Group A) or interrupted (Group B) and absorbable (polydioxanone) or nonabsorbable (prolene or polypropylene), 5-0, 6-0 or 7-0 sutures are used for end-to-end ductal anastomosis in patients undergoing biliary reconstructions.

\section{T-Tube:}

The advantage of biliary drainage is limitation of the inflammation and fibrosis may occur after the surgical procedure. In some authors' opinions, the presence of the biliary tube prevents anastomosis stricture.

\section{1-External T-drainage:}

It involves using a typical T-tube with insertion of its short branches into the bile duct and conducting of its long branch through the abdominal wall outside. It can be removed after healing of the endto-end ductal anastomosis (Fig. 1).

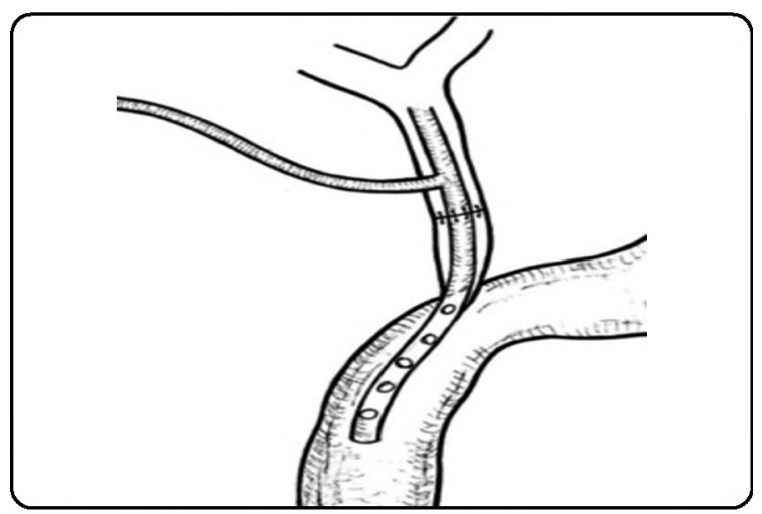

Fig. (1): T-Tube external biliary drainage.

\section{2- Internal Y-drainage:}

It involves insertion of short branches of the T-tube into both the right and left hepatic ducts, splinting of the anastomosis and conducting of its long branch into the duodenum by the papilla of Vater (Fig. 2).

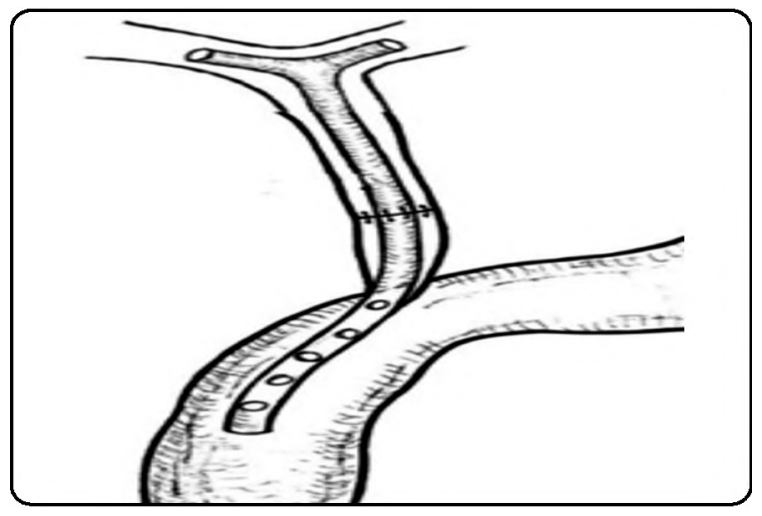

Fig. (2): T-Tube internal biliary drainage.

This drainage tube can be removed endoscopically after healing of the end-to-end ductal anastomosis. It should be noted that the internal Y-drainage is less traumatic than the external T-drainage. Therefore, it should be recommended as the drainage of choice in end-to-end ductal anastomosis.

\section{Results}

Comparable results of both surgical techniques; shows no difference in biliary complications, patient survival between the two analyzed groups.

The non-traumatic, mono-filament, interrupted 5-0 suture is the technique of choice for end-toend ductal anastomosis in patients with biliary duct injuries.

An anastomotic biliary leakage and bile duct stenosis are the common post-operative complications after end-to-end ductal anastomosis. In patients with end-to-end ductal anastomosis; endoscopic control and treatment of these complications are possible.

In anastomotic leakages and strictures, Endoscopic Retrograde Cholangio-Pancreatography (ERCP) with stenting or stricture balloon dilatation is the first-line treatment. Percutaneous transhepatic biliary drainage can also be performed.

The surgical techniques used in treating thirty patients with biliary disease requiring biliary reconstruction over a 36 month period are shown in (Table 1).

The median length of hospital stay was 7 (515) days. The median follow-up was 23 (2-36) months. Six patients were lost to follow-up. Two demented patients were discharged from followup at 6 months. Two (12.5\%) patients developed a recurrent Jaundice and Elevated liver enzymes at 6 and 16 months. Both were re-valuated by MRI Cholangiography for stenosis and show free biliary passage with no definite stenosis. 
Table (1): Characteristics of the study groups.

\begin{tabular}{|c|c|c|c|}
\hline Point of difference & Group A & Group B & $p$-value \\
\hline Number & 15 & 15 & \\
\hline Gender & $\begin{array}{l}\text { M: } 9(75 \%) \\
\text { F: } 6(25 \%)\end{array}$ & $\begin{array}{l}\text { M: } 11(91.6 \%) ; \\
F: 4(8.3 \%)\end{array}$ & \\
\hline Approach & $\begin{array}{l}\text { Continuous } \\
\text { Suture } \\
\text { Technique } \\
15(100 \%)\end{array}$ & $\begin{array}{l}\text { Interrupted } \\
\text { Suture } \\
\text { Technique } \\
15(100 \%)\end{array}$ & \\
\hline Conversions rate & $2(12.5 \%)$ & 0 & $p=0.004$ \\
\hline $\begin{array}{l}\text { Operative time } \\
\text { (minutes) }\end{array}$ & $160( \pm 140 \mathrm{~min})$ & $150( \pm 145 \mathrm{~m})$ & $p=0.004$ \\
\hline $\begin{array}{l}\text { Estimated bleeding } \\
(\mathrm{mL})\end{array}$ & $14.7(0-100)$ & $14.7(0-100)$ & $p=0.1 \mathrm{int}$ \\
\hline Hospital stay (days) & $7(5-15)$ & $7(5-15)$ & $p=0.08$ \\
\hline Incidence of Leakage & $0(0 \%)$ & $2(12.5 \%)$ & $p=0.1$ \\
\hline $\begin{array}{l}\text { Incidence of } \\
\text { re-stenosis }\end{array}$ & $2(12.5)$ & 0 & $p=0.1$ \\
\hline
\end{tabular}

Thirteen patients ( 6 males 7 females, average age 42 years, range 28-49) underwent Continuous sutures stentless End-to-end biliary reconstruction procedure with a mortality rate of $1.4 \%$, morbidity of $0 \%$ and recurrence rate at 2 years of $1.5 \%$. No worsening complications were recorded. General health well-being improvement was recorded in $97 \%$ of patients. The follow-up ranged between 6 and 12 months, there was no mortality (Table 2).

Table (2): Complications and outcome of study groups.

\begin{tabular}{llll}
\hline Complication & $\begin{array}{c}\text { Number of } \\
\text { patients (\%) } \\
\text { (Group A) }\end{array}$ & $\begin{array}{c}\text { Number of } \\
\text { patients (\%) } \\
\text { (Group B) }\end{array}$ & $p$-value \\
\hline Post-operative Cholangitis & $0 \%$ & $1(1.5 \%)$ & $p=0.1$ \\
Stricture & $1(1.5 \%)$ & $1(1.5 \%)$ & $p=0.1$ \\
Biliary Leakage & 0 & $1(1.5 \%)$ & $p=0.08$ \\
Wound infection & $0 \%$ & $1(1.5 \%)$ & $p=0.852$ \\
Chest infection & $2(2.5 \%)$ & $2(2.5 \%)$ & $p=0.852$ \\
Incisional Hernia & $0 \%$ & $3(4 \%)$ & $p=0.852$ \\
Mortality Rate & $0 \%$ & $0 \%$ & \\
\hline
\end{tabular}

The most complications were recorded: Biliary leakage, wound sepsis and subsequent incisional hernia and post-operative cholangitis. There were no Technique related complications. There was one conversion $(1.5 \%)$ for widespread abdominal adhesions following a previous surgery as shown in (Table 2).

1 of the 3 patients with bile leak were aged $<60$ years. All of them anastomoses performed above the level of the biliary confluence developed a leak, compared to $12(75.5 \%)$ of 15 anastomoses performed below the level of the biliary confluence. Pre-operative stenting was not a risk factor for bile leak in this series $(2.6 \%$ versus $6.1 \% ; p=0.13)$.
However, multi-variable stepwise logistic regression analysis showed that only biliary reconstruction following biliary injury (odds ratio $[\mathrm{OR}]=$ 6.84; $p=0.002$ ) and anastomosis above the biliary confluence $(\mathrm{OR}=4.62 ; p=0.03)$ were significant risk factors for biliary leak. Patients who underwent biliary reconstruction for injury had a six-fold higher risk of biliary leak compared to the rest of the cohort, while those who had an anastomosis above the confluence had a four-fold higher risk.

Our meta-analysis showed no significant difference in the outcome rate between continuous and rupted suture stent less biliary reconstruction techniques and 0.934; 95 percent CI, 0.457-1.910; $\mathrm{Z}$-value $=-0.187 ; p=0.852$ ) using random effect model.

Finally, our meta-analysis showed no statistical significance regarding Biliary leakage or stenosis between the two techniques (OR, 1.641; 95 percent CI, 0.547-4.926; $\mathrm{Z}$-value $=0.833 ; p=0.377$ ) using random effect modelling.

\section{Discussion}

Kasahara and colleages compared different biliary reconstructions in 321 recipients of right lobe LDLT. Biliary reconstruction was performed with $\mathrm{HJ}$ in 121 patients, end-to-end ductal anastomosis in 192 patients, and combined HJ and endto-end ductal anastomosis in 8 patients. They found that end-to-end ductal anastomosis showed a significantly lower incidence of leakage and a higher incidence of stricture [22].

Gazzaniga and colleagues performed end-toend ductal anastomosis in the immediate repair procedures only when the injury did not exceed one-third of the duct circumference and was not located more than $2 \mathrm{~cm}$ below the ductal confluence, or when injury was detected during the primary operation. In this series, injuries were type E2 in 18 patients, type E3 in 29 patients, and type E4 in 15 patients. Direct repair is not recommended when more than one-third of the bile duct circumference is injured. It cannot be carried out when the lesion involves the bifurcation of 1 or both hepatic ducts (Strasberg E3/E4). In such cases a Roux-en-YHepatico jejunostomy is the only procedure available to repair the damage [23].

Koffron and colleagues reported an associated injury of the artery in $61 \%$ of patients with recurrent strictures after primary bile duct repair [24]

Schmidt and colleagues reported that the presence of combined vascular and bile duct injuries 
and injury at or above the level of the biliary bifurcation were significant independent predictors of poor outcome in patients undergoing Roux-enY Hepatico -jejunostomy.

The advantage of biliary drainage is to limit the inflammation and fibrosis that occur after the surgical procedure. Therefore, some authors believe that the presence of the biliary tube prevents anastomosis stricture [25].

Scatton and colleagues compared the incidence of biliary complications after liver transplantation in patients undergoing end-to-end ductal anastomosis with or without T-tube in a large multicenter, prospective, randomized trial. The study included 108 patients divided into 2 groups: patients with $(n=90)$ or without $(n=90)$ a T-tube who underwent surgery in 6 French liver transplantation centers [26].

Continuous sutures are quicker to perform than Interrupted Suture, and the patients without T-tubes had fewer episodes of cholangitis and peritonitis, and they demonstrated a favorable trend for fewer overall biliary complications.

\section{Conclusion:}

End-to-end ductal anastomosis should be considered as the biliary reconstruction of choice because it is more physiologic than Hepaticojejunostomy and it is associated with fewer early post-operative complications.

Anastomosis of the common hepatic duct or common bile duct to a loop of jejunum (usually a Roux loop) with a single layer of monofilament absorbable sutures is now the standard technique of biliary-Enteric anastomosis in most centers. Anastomotic biliary leaks and strictures remain significant, though uncommon, complications.

End-to-end ductal anastomosis is used for biliary reconstruction in patients undergoing liver transplantation and surgical repair of iatrogenic biliary injuries.

Concluded that complications in the T-tube stent biliary reconstruction were less severe and required less aggressive treatment than those in the non-T-tube techniques. The incidence of anastomotic strictures was higher in patients without T-tubes.

The patients without T-tubes had fewer episodes of cholangitis and peritonitis, and they demonstrated a favorable trend for fewer overall biliary complications, anastomotic biliary leaks or fistulas, end- to-end ductal anastomosis revisions, dilatation and stenting, hepatic artery thrombosis.

Our analysis showed that: A bile duct injury (usually iatrogenic), reconstruction is performed on a non-dilated biliary tree in the presence of inflammation, complex damage to the duct, often inflicted by diathermy, and associated vascular injury. We speculate that this is the reason why these patients suffer a higher incidence of anastomotic leak. The biliary tree above the confluence gets progressively narrower and thinner, making anastomosis to these ducts technically more challenging.

\section{References}

1- SONDENAA K., KUBOTA K., SANO K., TAKAYAMA T. and MAKUUCHI M.: Successful reconstruction of segmental or subsegmental bile ducts after resection of hilar bile ducts: Short- and long-term results. Hepatogastro-enterology, 51: 1278-81, 2014.

2- JABLONSKA B., LAMPE P., OLAKOWSKI M., et al.: Hepatico -jejunostomy vs. end-to-end biliary reconstructions in the treatment of iatrogenic bile duct injuries. J. Gastro-intest Surg., 13: 1084-93, 2019.

3- STRASBERG S.M. and HELTON W.S.: An analytical review of vasculobiliary injury in laparoscopic and open cholecystectomy. HPB (Oxford), 13: 1-14, 2011.

4- JABLONSKA B., OLAKOWSKI M., LAMPE P., et al.: Quality-of-life assessment in the treatment of iatrogenic bile duct injuries: Hepatico-jejunostomy versus end-toend biliary reconstructions. ANZ J. Surg., 82: 923-7, 2012.

5- KASAHARA M., EGAWA H., TAKADA Y., et al.: Biliary reconstruction in right lobe living-donor liver transplantation: Comparison of different techniques in 321 recipients. Ann Surg., 243: 559-66, 2013.

6- KASAHARA M., EGAWA H., TAKADA Y., et al.: Biliary reconstruction in right lobe living-donor liver transplantation: Comparison of different techniques in 321 recipients. Ann. Surg., 243: 559-66, 2009.

7- LOPEZ R.R., BENNER K.G., IVANCEV K., KEEFFE E.B., DEVENEY C.W. and PINSON C.W.: Management of biliary complications after liver transplantation. Am. J. Surg., 163: 519-524, 1992.

8- RINGE B., OLDHAFER K., BUNZENDAHL H., BECHSTEIN W.O., KOTZERKE J. and PICHLMAYR R.: Analysis of biliary complications following orthotopic liver transplantation. Transplant Proc., 21: 2472-2476, 2009.

9- JABLONSKA B. and LAMPE P.: Reconstructive biliary surgery in the treatment of iatrogenic bile duct injuries. In: Brzozowski T., editor. New advances in the basic and clinical gastroenterology. Rijeka (HR): In. Tech., pp. 477494, 2012.

10- CASTALDO E.T., PINSON C.W., FEURER I.D., et al.: Continuous versus interrupted suture for end-to-end biliary anastomosis during liver transplantation gives equal results. Liver Transpl., 13: 234-8, 2012. 
11- DAVIDSON B.R., RAI R., KURZAWINSKI T.R., SELVES L., FAROUK M., DOOLEY J.S., et al.: Prospective randomized trial of end-to-end versus side-toside biliary reconstruction after orthotopic liver transplantation. Br. J. Surg., 86: 447-452, 2009.

12- RABKIN J.M., ORLOFF S.L., REED M.H., WHEELER L.J., CORLESS C.L., BENNER K.G., et al.: Biliary complications of side-to-side without $\mathrm{T}$ tube versus endto-end with or without $\mathrm{T}$ tube choledocho-choledochostomy in liver transplant recipients. Transplantation, 65 : 193-199, 2013.

13- NEUHAUS P., BLUMHARDT G., BECHSTEIN W.O., et al.: Technique and results of biliary reconstruction using side-to-side choledocho-choledochostomy in 300 orthotopic liver transplants. Ann. Surg., 219: 426-34, 2014.

14- WANG S.F., HUANG Z.Y. and CHEN X.P.: Biliary complications after living donor liver transplantation. Liver Transpl., 17: 1127-36, 2017.
15-KASAHARA M., EGAWA H., TAKADA Y., et al.: Biliary reconstruction in right lobe living-donor liver transplantation: Comparison of different techniques in 321 recipients. Ann. Surg., 243: 559-66, 2016.

16- GAZZANIGA G.M., FILAURO M. and MORI L.: Surgical treatment of iatrogenic lesions of the proximal common bile duct. World J. Surg., 25: 1254-9, 2011.

17- KOFFRON A., FERRARIO M., PARSONS W., et al.: Failed primary management of iatrogenic biliary injury: Incidence and significance of concomitant hepatic arterial disruption. Surgery, 130: 722-8, 2001.

18- SCHMIDT S.C., LANGREHR J.M., HINTZE R.E., et al.: Long-term results and risk factors influencing outcome of major bile duct injuries following cholecystectomy. Br. J. Surg., 92: 76-82, 2015.

19- SCATTON O., MEUNIER B., CHERQUI D., et al.: Randomized trial of choledocho-choledochostomy with or without a $\mathrm{T}$ tube in orthotopic liver transplantation. Ann. Surg., 233: 432-7, 2001

\section{دراسة مقارنة تقنية عمل وصلات القنوات المرارية وذالك باستخدام الفرز المنفصلة

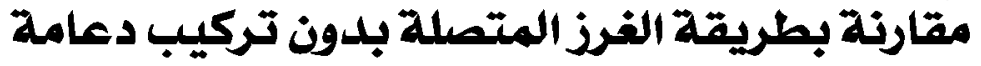

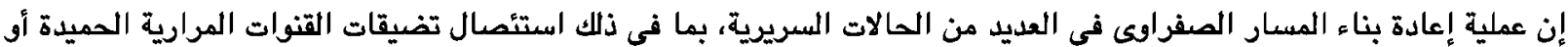

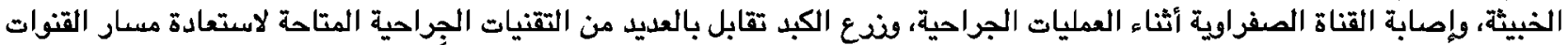

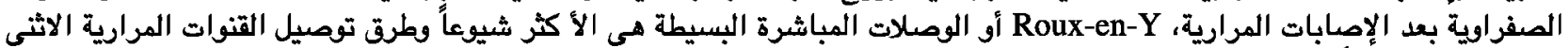

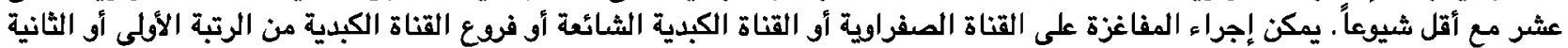

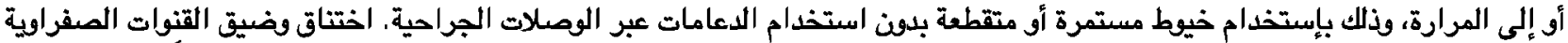

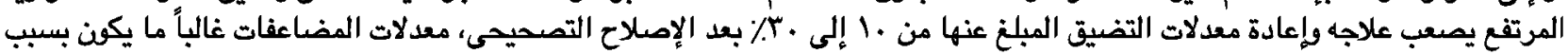
الإصابة بنقص الترية والتوتر فى موقع المفاغرة.

موضوع البحث: دراسة مشتركة مستقبلة ذات اثر رجعى لتقييم نتائج معدلات إعادة بناء القنوات الصفراوية باستخدام تقنية الخياطة المستمرة أو المتقطعة.

الهدف من الجحث: تقديم تحد تقنى جراحى لجراحى الكبد الصفراوى ودراسة المصابين المحتملين بأثر رجعى لتقييم نتائج المرضى والطرق.

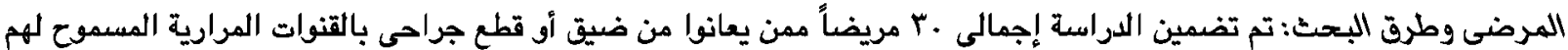

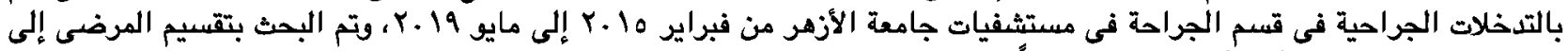

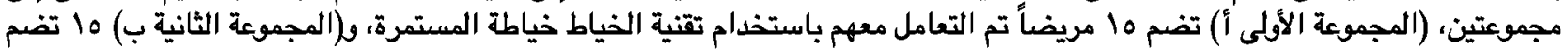

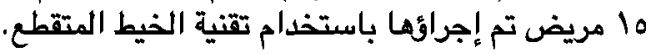

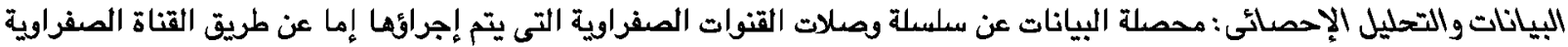

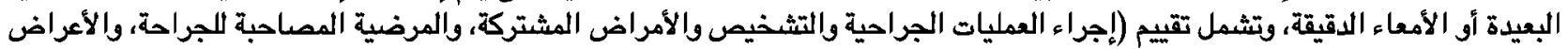

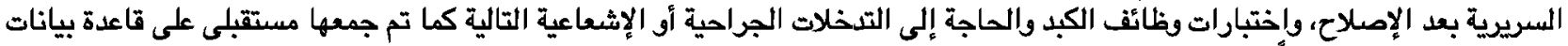
إلكترونية معتمدة مؤسسياً. تم وصف البيانات غير المعلمية على أنها بيانات متوسطة وواسعة النطاق وبيانات متوسطة

النتائج: تم إجراء إعادة بناء القنوات الصفراوية من النهاية إلى النهاية بين القناة الصفراوية أو القناة الصفراوية الكبدية مع الإمعاء الرفيعة.

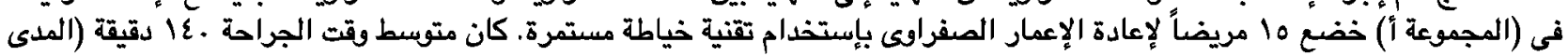

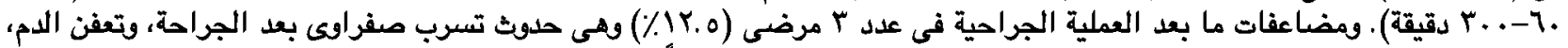

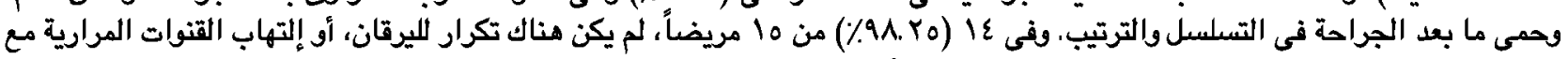
وظائف الكبد لون الحاجة إلى مزيد من الاجراءات الجراحية أو الإشعالِية. 